\section{An Image-Splitting Microscope for Accurate Measurement}

AN instrument has been devised for the very precise measurement of small objects, such as thin wires, blood cells or the like. The principle involved, which is a century and a half old, is that of the heliometer. The image is split into two complete images which may be sheared across each other by operation of a suitable control. The two images of the object are set edge to edge, an adjustment which is capable of great accuracy, and the amount of shear involved is read off from a suitable scale. The shear is then reversed, crossing the two images over and setting them edge to edge once more. The difference between the two readings of shear is proportional to the diameter of the object and can be calibrated by means of a 'stage micrometer'.

In the model which has been constructed, the shear is effected in an attachment to the eyepiece. This contains two prism blocks which form an optical system similar to that of a Mach-Zehnder interferometer. The two blocks are rotated with respect to each other about a vertical axis by means of a micrometer drive, giving a shear which is strictly proportional to the micrometer reading. Fig. 1 is a photograph of a blood smear, showing a few cells which have been sheared into edge-to-edge contact,

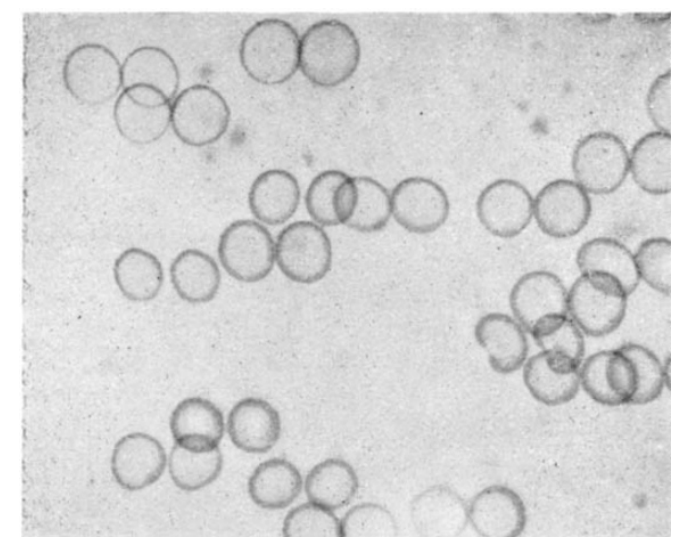
Fig. 1. Human blood cells under image-splitting microscope

and others which are not in contact or which overlap.

Using a $4 \mathrm{~mm}$. objective and a $15 \times$ eyepiece, repeated settings can be made on a single cell with a standard deviation of one or two micro-inches, that is, about 0.5 per cent of the diameter of the red cells.

Objects of a periodic nature, such as wire helices, can be very rapidly checked for pitch errors by shearing the images of corresponding elements of structure in successive periods into coincidence and passing the object longitudinally under the microscope. Small pitch errors are then immediately evident to the eye and can be measured precisely when detected.

As the only modification to the microscope is in the eyepiece, no restriction is placed on the method of microscopy or the powers used.

\section{J. Dyson}

Associated Electrical Industries Ltd.,

Research Laboratory,

Aldermaston Court, Berkshire.

Sept. 23.

\section{CHEMISTRY}

\section{Evidence for a Hydrate of Cellulose from Studies of its Surface Properties}

DURING an investigation of the surface properties of cellulose the relationship between the contact angle of water on cellulose and the water content of the cellulose was determined. The determination of the water-cellulose-air contact angle was done with the sessile drop method on regenerated cellulose films, and with the vertical rod method using thick cellulose fibres. (The model filaments of P. H. Hermans ${ }^{1}$ ). The contact angle of water on cellulose was determined at $20^{\circ} \mathrm{C}$. in a gas phase consisting of air with a relative humidity with which the cellulose films and fibres of different water content were in equilibrium. Changes in the water content of the cellulose, ranging from 0 t.o 25.9 per cent absorption, were obtained by keeping the cellulose at $20^{\circ} \mathrm{C}$. in air of different relative humidities varying from 0 to 95 per cent.

The water-cellulose-air contact angle was found to vary as a function of the water absorption of the cellulose, or the relative humidity of the air, as shown in Fig. 1. The numbers on the curve refer to the relative humidities with which the cellulose was in equilibrium at the various water absorptions.

From this contact angle-water absorption relationship it can be seen that the contact angle is almost constant from nil to about 12 per cent water absorption, whereas there is a sharp decrease in the contact angle when the water absorption of the cellulose increases from 12 to 25.9 per cent. This shows that when the relative humidity of the air is increased from 0 to 95 per cent, the first 12 per cent water absorption has a negligible effect on the surface properties of cellulose, but a further 13.9 per cent water absorption causes the contact angle to be reduced from $24 \cdot 4^{\circ}$ to $13 \cdot 4^{\circ}$.

The hydrophilic character of cellulose, as revealed by the water-cellulose-air contact angle, is governed by the number, the steric arrangement and the accessibility of its hydroxyl groups. Probably because of its submicroscopic crystalline structure a certain number of its hydroxyl groups are not accessible to water, and its hydrophilic character was found to be less than expected. Water absorption by cellulose, which causes its total number of hydroxyl groups to increase, ought therefore to increase its hydrophilic character and to reduce its contact angle with water. That cellulose could take up about 12 per cent water with only a negligible effect on its surface properties, indicates that this amount of water is firmly bound to

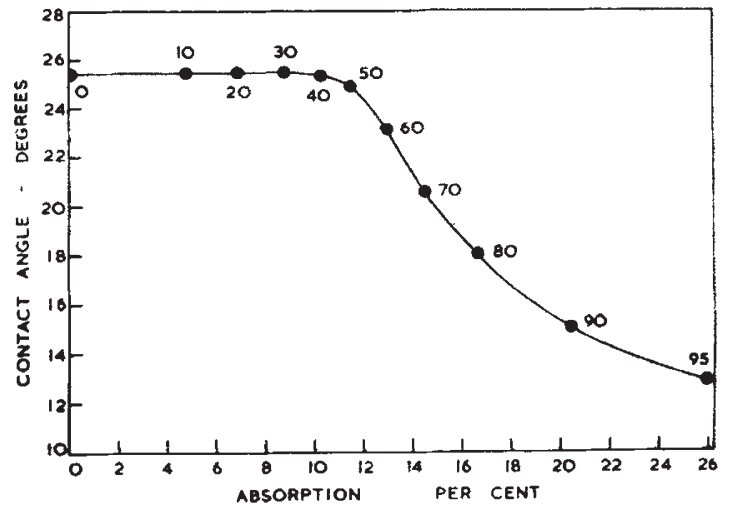

Fig. 1 